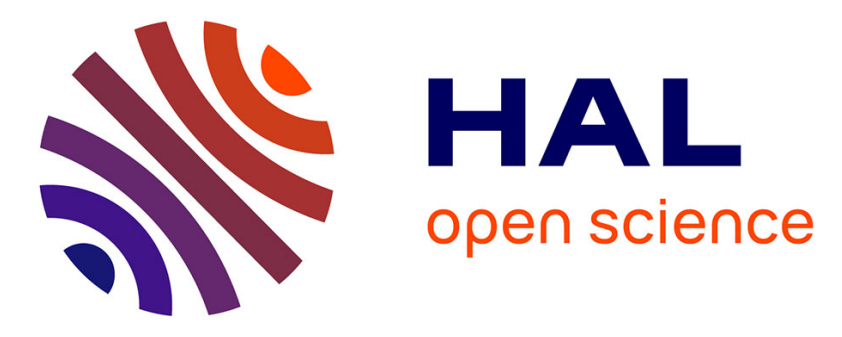

\title{
L'attraction des universités des villes nouvelles franciliennes
}

\author{
Nadine Cattan, Sandrine Berroir, Thérèse Saint-Julien
}

\section{To cite this version:}

Nadine Cattan, Sandrine Berroir, Thérèse Saint-Julien. L'attraction des universités des villes nouvelles franciliennes. Les Annales de la Recherche Urbaine, 2005, 98, pp.67-73. halshs-00152888

\section{HAL Id: halshs-00152888 \\ https://shs.hal.science/halshs-00152888}

Submitted on 7 Jun 2007

HAL is a multi-disciplinary open access archive for the deposit and dissemination of scientific research documents, whether they are published or not. The documents may come from teaching and research institutions in France or abroad, or from public or private research centers.
L'archive ouverte pluridisciplinaire HAL, est destinée au dépôt et à la diffusion de documents scientifiques de niveau recherche, publiés ou non, émanant des établissements d'enseignement et de recherche français ou étrangers, des laboratoires publics ou privés. 


\title{
L'ATTRACTION DES UNIVERSITES DES VILLES NOUVELLES FRANCILIENNES
}

\author{
Sandrine Berroir, Nadine Cattan, Thérèse Saint-Julien \\ UMR Géographie-cités \\ 13 rue du four - 75006 Paris
}

\section{Introduction}

Le projet des villes nouvelles en Ile-de-France s’est construit sur la volonté politique de créer de véritables centres urbains, susceptibles de contrecarrer la très longue domination de Paris. La décision d'implanter dans ces villes des équipements de niveau supérieur comme les universités s'est inscrite dans cette perspective de réaménagement de la centralité régionale et d'appui au développement de centres capables d'attraction et de diffusion à l'échelle de l'ensemble de la région. Les universités, par l'attractivité qu'elles sont censées susciter tant auprès des populations que des entreprises, par les réseaux qu'elles créent, par les images qu'elles véhiculent, sont devenues un enjeu prioritaire des stratégies de développement des villes et des régions métropolitaines. Elles constituent aujourd'hui un vecteur incontournable de la centralité urbaine. Cette étude a pour objet d'identifier la contribution des universités au rayonnement des villes nouvelles. Elle s'appuie sur les formes de l'inscription spatiale des attractivités de ces établissements dans le système francilien de l'offre et de la demande universitaire $^{1}$ (Berroir, Cattan, Saint-Julien, 2005).

A la rentrée universitaire 2002, un peu plus de $10 \%$ des étudiants franciliens inscrits à l'université ${ }^{2}$ le sont dans un établissement localisé en ville nouvelle (soit environ 54300 étudiants). Leur relative concentration dans les premiers cycles de formation donne une certaine spécificité à ces universités dans le paysage universitaire francilien. La relative spécialisation de leurs diplômes, résultat d'une offre de formation à finalité fortement professionnelle, accentue l'identité de ces universités nouvelles de la grande couronne, ce que l'étude de l'Ofipe (2005, p. 4) a très clairement pointé : " près d'un étudiant sur trois au niveau licence, plus de $70 \%$ au niveau bac + cinq sont inscrits dans des formations professionnelles » ce qui distingue d'emblée les universités des villes nouvelles, et en particulier Evry et Marne-la-Vallée, des autres universités françaises en général, et des autres universités franciliennes en particulier..

Les capacités d'attraction des universités des villes nouvelles résultent, pour partie, de cette offre de formation spécifique. L’étendue et les formes des aires de recrutement des pôles universitaires, appréhendées par les navettes entre le lieu de domicile et le lieu d'étude, donnent un premier éclairage sur la manière dont ces pôles organisent leur environnement et captent à leur avantage des parts du «marché estudiantin » francilien. La participation aux

\footnotetext{
${ }^{1}$ Données mises à disposition, par la Direction de l’Evaluation et de la Prospective : base de données des étudiants inscrits dans les universités franciliennes, aux deux rentrées universitaires de 2001 et 2002.

${ }^{2}$ On considère chaque établissement comme un tout. La localisation de celui-ci est celle du siège de l'université, sur lequel l'ensemble des effectifs étudiants est comptabilisé. Ainsi, les antennes ne sont pas prises en compte en tant que telles. L'étude ne permet donc pas d'identifier les attractions de ces dernières.
} 
réseaux de la mobilité interuniversitaire, évaluée par les changements d'établissements en cours d'études, est une deuxième composante forte de l'attractivité d'un pôle universitaire. Elle permet de déterminer, à chaque niveau d'études, la place des établissements dans le jeu de la concurrence et de la complémentarité qui structure l'offre de formation en Ile-de-France. La dernière dimension de cette attraction, celle de longue portée, est mesurée par l'accueil d'étudiants étrangers.

\section{Le proche et le lointain dans le recrutement}

Eléments structurants de l'espace régional, les centres universitaires sont, en premier lieu, des pôles d'attraction qui drainent la demande locale et construisent des liens préférentiels avec certains territoires. $40 \%$ des étudiants qui résident en petite ou grande couronne y étudient également. Cette proportion est faible comparativement au $78 \%$ des étudiants qui, résidant à Paris, fréquentent un établissement parisien. Par ailleurs, près des deux-tiers de la population estudiantine résidant en périphérie alimentent les établissements parisiens, tandis que près d'un quart seulement des étudiants résidant à Paris alimente les universités périphériques. Audelà de cette image très synthétique et très macro géographique des concurrences entre universités, laquelle témoigne d'un certain maintien de la dépendance des périphéries régionales vis-à-vis du « pôle parisien », on constate que les universités de la grande couronne ont des aires de recrutement bien circonscrites. Celles-ci reposent sur deux dimensions complémentaires. La première est relative à la capacité de polarisation d'une université, qui dit sa force du rayonnement : chacune attire les étudiants en provenance d'un certain nombre de communes, lesquelles qui constituent son aire d'attraction, ou encore son bassin de recrutement. Plus le nombre des étudiants attirés est grand et plus le bassin est étendu, plus l'attraction de l'établissement est forte. La seconde dimension concerne le degré de l'emprise qu'une université exerce en un lieu, relativement à celle qu'impriment en ce lieu les universités concurrentes. Cette dimension définit les dépendances territoriales engendrées localement par chacun des centres universitaires. Certes, l'ombre d'une carte universitaire plane sur cet exercice. Selon les disciplines, les universités, mais aussi selon les années, les inscriptions des étudiants sont soumises à des règles de sectorisation territoriale. Cette sectorisation n'est pas explicitement prise en compte. Seuls ses effets en sont intégrés dans les part du marché régional de la demande de formation que les villes nouvelles réussissent à capter.

\section{Les inégales attractions de proximité}

Les attractions des universités se structurent sous la forme de gradients qui correspondent à la variation de la proportion d'étudiants qui fréquentent un établissement donné, en fonction de la distance résidence/étude ${ }^{3}$. Ces gradients sont un bon révélateur du positionnement des universités des villes nouvelles dans le système des polarisations universitaires régionales. Ils font clairement apparaître l’originalité des profils des universités de la grande couronne.

Les gradients de polarisation des universités dont le siège est parisien sont relativement réguliers et de pente assez forte (figure 1), ce qui signifie que ces établissements recrutent pour l'essentiel sur d'assez courtes distances : près d'un tiers des étudiants qui les fréquentent

\footnotetext{
${ }^{3}$ Un étudiant est mobile quand sa commune de résidence est différente de la commune du siège de l’Université.
} 
résident à moins de 5km, entre les deux-tiers et les trois-quarts à moins de $10 \mathrm{~km}$. Au-delà, l'apport s'assèche rapidement. Les universités de la petite couronne (Paris 8-Saint-Denis, Paris 10-Nanterre, Paris 12-Créteil Val de Marne, Paris 13-Villetaneuse), apparemment prises en tenaille entre les établissements parisiens et ceux de la grande couronne, tirent bien leur épingle du jeu. Alimentées, à hauteur de 20\%, par des étudiants résidant à Paris, leurs gradients de polarisation sont très ressemblants, tant ils sont façonnés par leur situation relative vis-à-vis du pôle central. Ils passent tous par un maximum de concentration à la hauteur de Paris, entre 5 et $20 \mathrm{~km}$.

Comparativement, les universités de la grande couronne ont des gradients de polarisation plus différenciés : ils dépendent, à la fois, de la distance à Paris et de leur localisation dans un secteur particulier de la région (figure 1). Marne-la-Vallée et Evry ont un gradient dont la pente est assez régulière, avec une diminution très lente jusqu'au seuil de $20 \mathrm{~km}$ et plus rapide ensuite. Avec près de $30 \%$ d'étudiants qui résident à moins de $5 \mathrm{~km}$, l'université de CergyPontoise semble, plus que les autres, tributaire d'un recrutement sur courte distance. Toutefois, avec plus de 30\% d'étudiants qui résident entre 10 et $20 \mathrm{~km}$, cette université puise également dans les ressources estudiantines parisiennes. A l'inverse, Versailles Saint-Quentin et Paris 11-Orsay, avec 16\% d'étudiants seulement qui viennent de leur périphérie immédiate, sont les deux universités dont le recrutement est relativement le moins local. On peut s'étonner de la ressemblance des gradients de ces deux universités du sud-ouest de la région que tout sépare, l’âge d'abord, presque quarante années séparent leur création, le projet initial et l'orientation disciplinaire. Un fort effet de secteur géographique semble les rapprocher et ce d'autant plus qu'avec un fort pic de concentration sur le centre de la région francilienne, ces deux universités empiètent sur les territoires des universités centrales. Paris 11-Orsay apparaît comme la plus parisienne de toutes les universités périphériques, en ce qu'elle capte plus de $50 \%$ de ses étudiants à Paris et dans la petite couronne. Université scientifique, elle est, de toutes les universités franciliennes, celle dont le bassin de recrutement local est relativement le plus faible.

Les formes des aires de polarisation sont particulièrement sensibles aux effets de cycle. Au niveau du $3^{\mathrm{e}}$ cycle, les freins de la distance ne jouent presque plus. Les marges de liberté des étudiants face à la distance qui les séparent des différents établissements sont beaucoup plus grandes. Le marché régional des formations universitaires de $3^{\mathrm{e}}$ cycle paraît assez fortement intégré.

Au total, les formes de la polarisation, font apparaître, surtout pour le $3^{\mathrm{e}}$ cycle que CergyPontoise et Versailles-Saint-Quentin grignotent, plus que ne le font Evry et Marne-la-Vallée, des parts du marché parisien, intégrant ainsi pleinement le système concurrentiel régional.

\section{Des environnements bien « tenus »}

Dans l'appréciation de l'attractivité des universités, l'évaluation de l'intensité de leur emprise locale est un complément obligé. Cette emprise correspond aux dépendances territoriales que les établissements universitaires suscitent dans leur environnement. Evaluée pour chaque commune, par la proportion d'étudiants inscrits dans un établissement d'une ville nouvelle dans le total des étudiants résidant dans la commune, l'emprise tient compte explicitement des propriétés des lieux émetteurs et de leur force émettrice. Elle fournit une indication de l'adéquation entre l'offre et la demande de formation et des concurrences auxquelles se livrent dans la commune les différents établissements. 
Dans le contexte universitaire très dense et très concurrentiel qui caractérise la région Ile-deFrance, l'emprise locale de chacune des universités des villes nouvelles se construit de façon très dissymétrique (figure 2.a). Toutes choses égales quant à la distance, les communes sont d'autant plus dépendantes de l'établissement de la ville nouvelle, qu'elles se trouvent dans sa proximité, et qu'elles sont situées dans une direction diamétralement opposée à celle de Paris. Ces communes sont moins directement exposées à la concurrence très directe des grands établissements centraux. Sur le chemin «naturel» de la mobilité qui tend à mettre ces communes de la périphérie dans la dépendance du centre parisien principal, l’offre de formation des universités des villes nouvelles représente une opportunité réelle de satisfaction de la demande. Elle s'interpose donc efficacement pour retenir, à son avantage, une part de ce flux ascendant.

Toutefois, l'emprise spatiale de cette offre périphérique diffère tant par son intensité que par sa forme d'une ville nouvelle à une autre. Evry et Cergy-Pontoise tiennent relativement bien leur environnement immédiat, mais leur force relative de mise en dépendance décline régulièrement et parfois rapidement. Marne-La-Vallée et Versailles-Saint-Quentin, très concurrencées dans leur environnement proche, sont en revanche capables d'imposer de plus fortes dépendances à moyenne et à grande distances.

Par rapport à ce schéma général, les portées et de l'intensité des dépendances engendrées par les $3^{\mathrm{e}}$ cycle sont affaiblies (figure 2.b). C'est plus particulièrement le cas autour de Marne-laVallée et d'Evry. L'affaiblissement des dépendances est un peu moins spectaculaire autour de Cergy-Pontoise et de Versailles-Saint-Quentin. La lecture est toutefois plus complexe qu'il n’y paraît, puisque Marne-la-Vallée et Versailles-Saint-Quentin exercent en outre, sur un petit nombre de communes dispersées dans la région, des taux de dépendance relativement élevés, ce qui pourrait indiquer que l'assise territoriale de ces deux universités s’inscrit dans un rayon plus large, qui va au-delà des limites de leur secteur « naturel » de rétention.

Globalement, les emprises spatiales que les universités des villes nouvelles impriment sur leur environnement sont fortes sur les courtes et moyennes distances et plus faibles ensuite. Tout se passe comme si les étudiants résidant dans le voisinage des villes nouvelles étaient relativement captifs de l'offre universitaire dispensée dans ces dernières. C'est comme si, à ce stade, les localisations périphériques protégeaient ces centres des concurrences externes. La force relative de l'emprise spatiale des universités des villes nouvelles s'affaiblit rapidement aux passages des cycles. Dans le jeu des concurrences intra-régionales, les universités des villes nouvelles sont pénalisées du fait de leur immersion dans un environnement de faibles densités et de certaines limites de leur offre de formation en $3^{\mathrm{e}}$ cycle.

\section{Les concurrences interuniversitaires}

Dans le jeu des concurrences que se livrent les établissements universitaires franciliens, les mobilités quotidiennes ne suffisent pas à qualifier leurs capacités d'attraction. Le positionnement de ces pôles tient aujourd'hui beaucoup à la mobilité interuniversitaire que les étudiants décident d'effectuer au cours de leurs études en changeant d'établissement. L'enjeu de ces échanges est, en effet, considérable puisque, en Ile-de-France, un étudiant sur dix change d'université en cours d'études et que cette mobilité est d'autant plus élevée que le niveau d'étude croît. Compte tenu de l'histoire, de la position et du rôle qui était assigné aux universités des villes nouvelles dès leur création, nous avons focalisé notre attention sur deux aspects particuliers des migrations interuniversitaires. On évalue, dans un premier temps, les 
relations que les établissements des villes nouvelles nouent avec ceux du centre parisien. On analyse ensuite les liens que ces établissements tissent avec les universités de la province.

\section{Quelles résistances face aux universités centrales ?}

En moyenne, tous cycles confondus, les étudiants des universités des villes nouvelles migrent, dans des proportions presque équivalentes, vers une université parisienne et vers une autre université de la petite ou de la grande couronnes (tableau 1). Cela signifie que les étudiants des établissements des villes nouvelles ne sont nécessairement condamné a une inscription dans une université centrale Les étudiants de Versailles-Saint-Quentin sont ceux qui apparaissent dépendre le plus de l'offre de formation parisienne, et ceux d'Evry ceux qui en sont le moins dépendants. Toutefois, à l'entrée en $3^{\mathrm{e}}$ cycle, Paris redevient la destination principale des étudiants émigrants de ces universités à hauteur de $55 \%$. Cette situation des universités nouvelles n'est pas spécifique, puisqu'au passage du $2^{\mathrm{e}}$ au $3^{\mathrm{e}}$ cycle, une proportion élevée des étudiants émigrants de Paris 11-Orsay et Paris 13-Villetaneuse retrouve aussi le chemin de Paris.

Les villes nouvelles captent à leur tour, tous cycles confondus, $10 \%$ des étudiants parisiens qui changent d'université au cours de leur cursus universitaire, et près de $12 \%$ de ceux pour lesquels la migration s'effectue entre le $2^{\mathrm{e}}$ et le $3^{\mathrm{e}}$ cycle. Cependant, ces universités des villes nouvelles dépendent moins des contingents parisiens que des universités comme Paris 11Orsay ou Paris 13-Villetaneuse (tableau 2). Pour les villes nouvelles, ces immigrants venant des universités de Paris centre représentent $43 \%$ des effectifs reçus tous cycles confondus, et $46 \%$ à l'entrée en $3^{\mathrm{e}}$ cycle. Pour Paris 11-Orsay ces taux s'élèvent respectivement à $59 \%$ et $63 \%$.

Dans ce jeu de l'émigration - immigration avec Paris, Evry tisse des connexions diversifiées dans le champ des universités de la périphérie construisant un réseau d'échanges relativement indépendant des universités centrales. A l'inverse, Versailles-Saint-Quentin semble se placer plus nettement dans l'ombre des réseaux parisiens. Cergy-Pontoise et Marne-la-Vallée sont quant à elles dans une situation intermédiaire.

\section{Quelle face à face avec les universités de province ?}

Parmi les étudiants qui changent d'université en cours d'études, 7800 quittent l’Ile-de-France pour poursuivre leurs études dans une université de province, soit $30 \%$ de l'ensemble des départs. Les étudiants des universités de la grande couronne (figure 3.a) sont en moyenne plus attirées par des universités de province que ne le sont les étudiants des universités centrales (à l'exception notable de Paris 3 et surtout, de Paris 6). Faut-il voir là un rejet plus fréquent du modèle de vie métropolitain de la part de jeunes gens ayant vécu, étant même souvent nés, dans les périphéries de l'agglomération parisienne? Doit-on invoquer une meilleure connaissance des offres de formation dispensées par les universités de province ? A moins qu'il ne s'agisse de filtrages à l'entrée moins contraignants que dans les universités centrales ? Seule un enquête menée sur les motivations effectives de ces migrations pourraient éclairer ces questions.

Ces flux vers la province sont très largement compensés par l'attractivité qu'exerce globalement l'ensemble des universités franciliennes sur les étudiants de province. Plus de 11000 étudiants quittent la province pour rejoindre un établissement universitaire francilien, soit 37\% des nouveaux inscrits dans un de ces derniers. Le solde des échanges, entre l'Ile-de- 
France et la province, est donc largement positif au profit des universités de l’Ile-de-France. Pourtant, les universités franciliennes sont à ce stade inégalement attractives (figure 3.b). Cette différence traverse les établissements des villes nouvelles. Evry et Versailles-SaintQuentin affichent un bilan très positif, la part des arrivées en provenance d'universités de province y étant supérieure à la moyenne de l'ensemble des universités de la région. A l'inverse, Marne-la-Vallée et Cergy-Pontoise sont relativement moins attractives pour un public étudiant non francilien.

On peut en conclure que les universités des villes nouvelles ont su fabriquer de solides centralités de proximité, répondant ainsi à la demande de formation des étudiants de la grande couronne. Elles ont su également se positionner dans le système des concurrences interuniversitaires, retenant certes mal leurs étudiants de $3^{\mathrm{e}}$ cycle mais sachant se montrer attractives pour des étudiants parisiens migrant en cours d'études. Leur force d'attraction sur les étudiants des universités des autres régions françaises sont relativement contrastées.

\section{L’ouverture internationale}

L'ouverture aux étudiants étrangers est une dernière composante, incontournable, de l'attractivité relative des universités des villes nouvelles, à longue portée cette fois. Elle est toutefois de mesure délicate. Sont comptabilisés ensemble les étudiants étrangers résidant en France, après y avoir effectué leur scolarité antérieure, et des étudiants dont la migration est directement en relation avec l'inscription dans une université de la région. Le mélange de ces deux populations demande une grande prudence quant à l'exploration de cette question. Cette limite étant posée, l'examen des disparités du rayonnement international des universités franciliennes est éclairant. En 2002, les étudiants étrangers représentent en moyenne près de $18 \%$ des effectifs des universités franciliennes. Ces proportions moyennes peuvent apparaître faibles, d'autant que, dans ces étudiants sont précisément comptabilisés tous ceux qui ont résidé en France durant tout ou partie de leur scolarité secondaire. Cet effet est probablement sensible pour Paris 8 - Saint-Denis, où 33\% des inscrits sont des étudiants étrangers. Pour tous les établissements, le taux moyen d'ouverture s'élève régulièrement de $12 \%$ en $1^{\mathrm{er}}$ cycle, à $18 \%$ en $2^{\mathrm{e}}$ cycle et enfin, à $27 \%$ en $3^{\mathrm{e}}$ cycle. Cependant, toutes les universités ne montrent pas les mêmes capacités d'ouverture. Si en $1^{\mathrm{er}}$ cycle, l'attrait exercé sur les étudiants étrangers par les universités de la grande couronne est en moyenne très faible et qu'il reste inférieur à la moyenne pour les deux autres cycles, le rattrapage que ces universités opèrent du $1^{\mathrm{er}}$ au $3^{\mathrm{e}}$ cycle est remarquable : la proportion des étudiants étrangers en troisième cycle y est en moyenne multipliée par 3,9 par rapport au premier cycle, contre 2,3 à Paris et 2,1 en petite couronne. Versailles Saint-Quentin arrive en tête des universités de ville nouvelle avec près de $14 \%$ d'étudiants étrangers. Ce taux est certes légèrement en dessous de la moyenne régionale, mais cette université opère une belle remontée à la hauteur du $3^{\mathrm{e}}$ cycle avec $30 \%$ d'étudiants étrangers inscrits. Evry occupe aussi une position très honorable avec respectivement $13 \%$ et $29 \%$. Pour l'accueil en $3^{\mathrm{e}}$ cycle, Marne-la-Vallée se défend bien avec $27 \%$ soit un niveau proche de la moyenne. Seule Cergy-Pontoise fait moins bien.

\section{Conclusion}

En plaçant la mobilité des étudiants au centre des questionnements, ce travail fournit une image originale des potentialités d'attraction et des capacités structurantes des pôles universitaires des villes nouvelles. Il montre que les offres de formations de ces pôles, relativement spécialisées et professionnalisantes, ont contribué à fidéliser une clientèle de proximité et à instaurer, à l'échelon des $3^{\mathrm{e}}$ cycles en particulier, des flux de mobilité 
quotidienne et de migrations interuniversitaires qui freinent l'amplification de la dissymétrie des échanges. Les polarisations que mettent en place les universités des villes nouvelles esquissent donc un schéma polycentrique de l'espace régional métropolitain. Ces mobilités permettent de nuancer l'image duale d'une offre universitaire encore marquée du sceau d'un déséquilibre, entre une concentration centrale d'universités de grande taille thématique, et plutôt généralistes, et d'un regroupement à la périphérie d'établissements de taille plutôt moyenne et à orientation relativement professionnelle. La dynamique de l'attractivité des universités localisées en ville nouvelle donne certains signes encourageants de la contribution des universités à la constitution de nouveaux centres urbains, ayant acquis certains moyens de leur autonomie.

Tableau 1 - Les destinations des étudiants des universités des villes nouvelles

Part des étudiants qui quittent une université dans le total des départs :

\begin{tabular}{|c|c|c|c|c|c|c|}
\hline \multirow{3}{*}{ Université d'origine } & \multicolumn{4}{|c|}{ Université de destination en : } & \multirow{2}{*}{\multicolumn{2}{|c|}{ Paris }} \\
\hline & \multicolumn{2}{|c|}{ Grande Couronne } & \multicolumn{2}{|c|}{ Petite Couronne } & & \\
\hline & tous cycles & 3e cycle & tous cycles & 3e cycle & tous cycles & 3e cycle \\
\hline Cergy-Pontoise & 16 & 19 & 36 & 20 & 48 & 61 \\
\hline Versailles-Saint-Quentin & 25 & 24 & 22 & 13 & 53 & 63 \\
\hline Evry-Val d'Essone & 27 & 26 & 32 & 33 & 41 & 41 \\
\hline Marne-la-Vallée & 14 & 27 & 40 & 23 & 46 & 50 \\
\hline Moyenne universités nouvelles & 20 & 23 & 32 & 22 & 48 & 55 \\
\hline Paris 11-Orsay & 20 & 17 & 20 & 13 & 60 & 70 \\
\hline Paris 13-Villetaneuse & 24 & 29 & 32 & 21 & 44 & 50 \\
\hline
\end{tabular}
source : $D E P, 2002$

Tableau 2 - Attractivité des établissements de la grande couronne vis-à-vis des universités parisiennes

\begin{tabular}{ccc}
\hline $\begin{array}{c}\text { Part des étudiants des universités parisiennes } \\
\text { dans le total des arrivées de : }\end{array}$ & Tous cycles & 3e cycle \\
\hline Cergy-Pontoise & 36 & 42 \\
Versailles-Saint-Quentin & 52 & 57 \\
Evry-Val d'Essonne & 39 & 38 \\
Marne-la-Vallée & 46 & 47 \\
Moyenne universités nouvelles & 43 & 46 \\
Paris 11-Orsay & 59 & 63 \\
Paris 13-Villetaneuse & 48 & 48 \\
\hline
\end{tabular}


Figure 1 - Les gradients de Pattraction

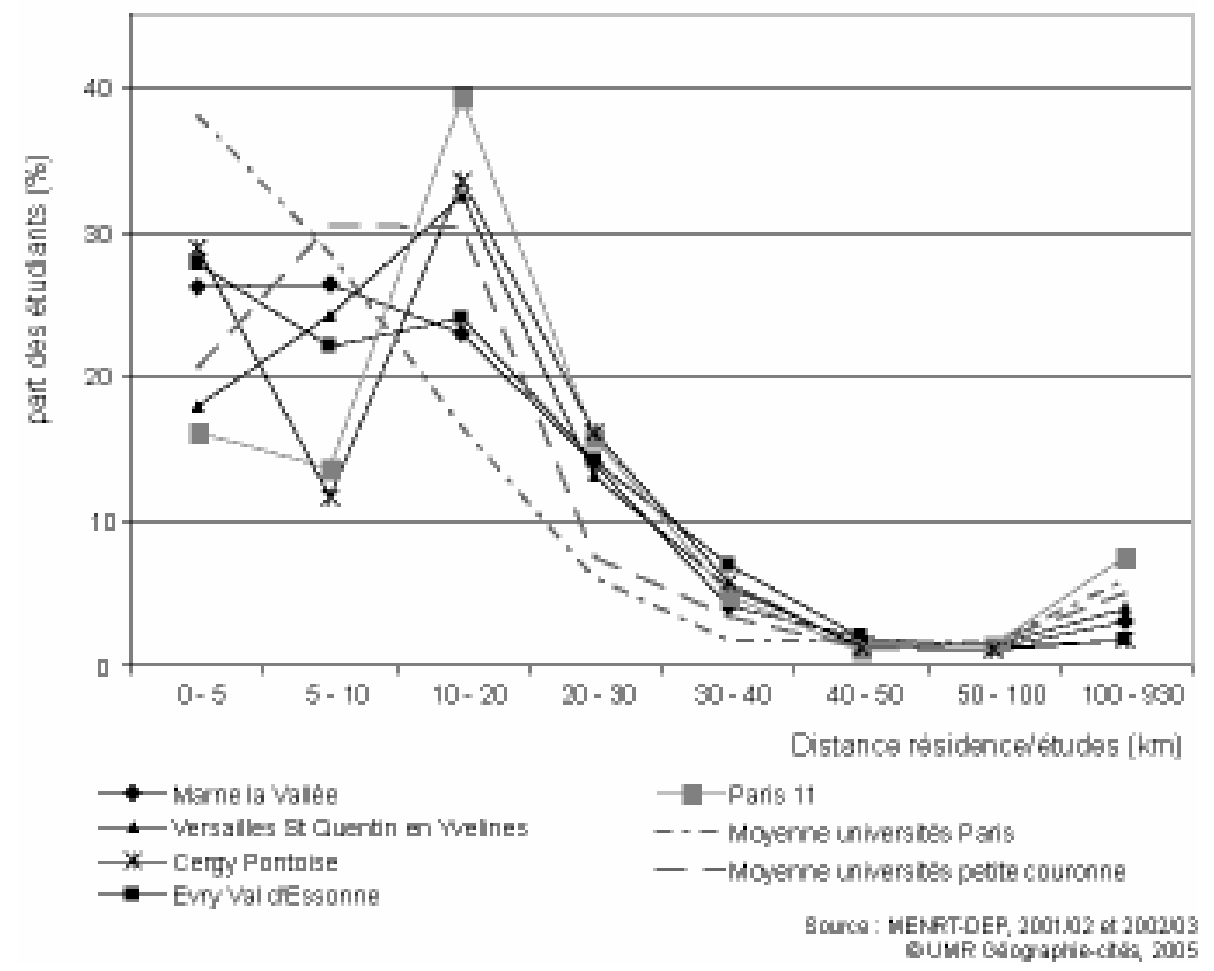


Figure 2 - Les aires de dépendance des universités des villes nouvelles
a. tous cycles
b. à l'entrée en 3ème cycle
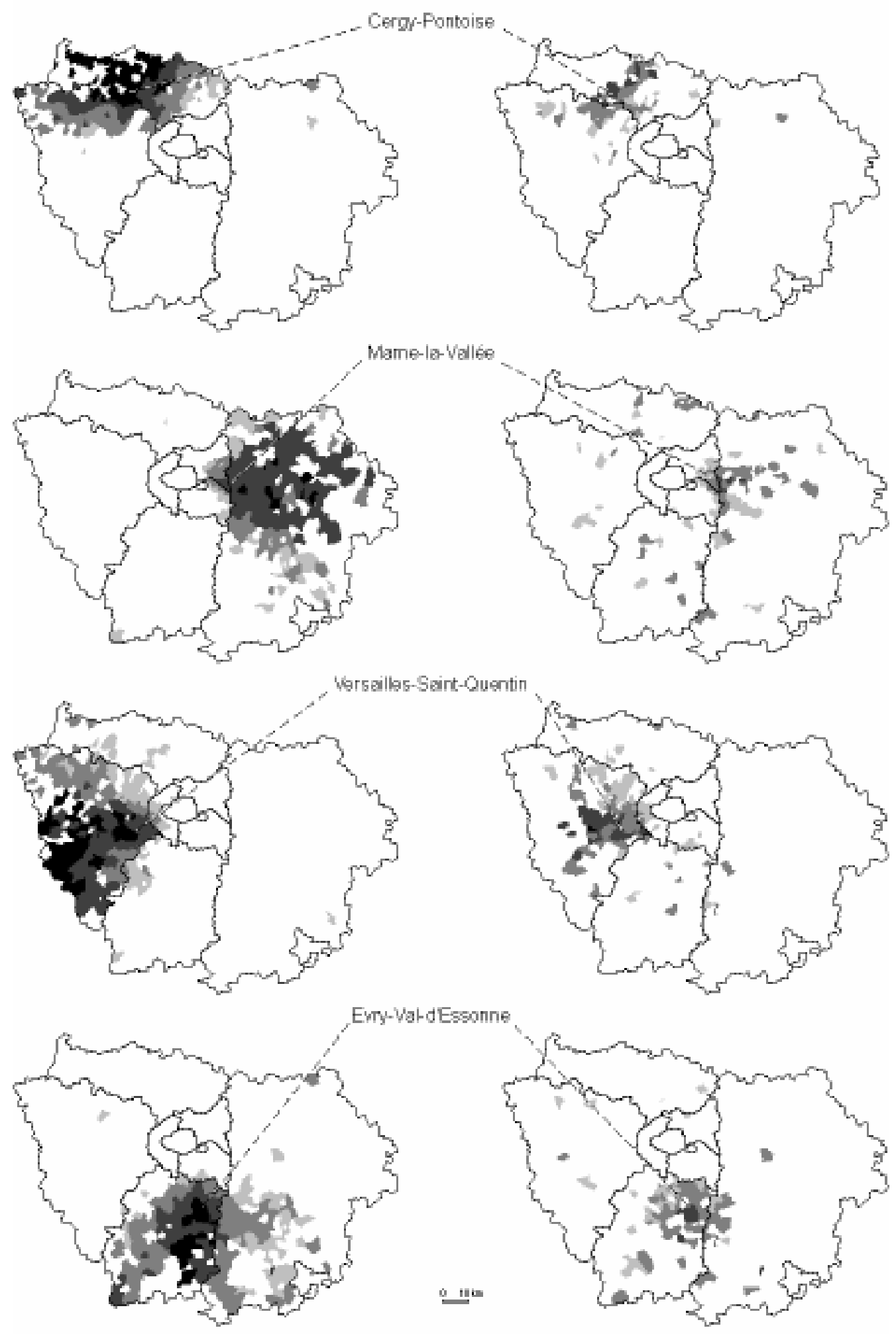

Taux de dependence $[\%]$

$\square>50$

25 a 50

$10 \triangle 25$

5810

× 5 ou moins de 5 inscrits dens une universite franclienne

Fat suec Pherarto- htp Operso club-internd tiphigeo 


\section{Figure 3 - Les universitaires Ile-de-France/province}

\section{a) Les départs vers la province}

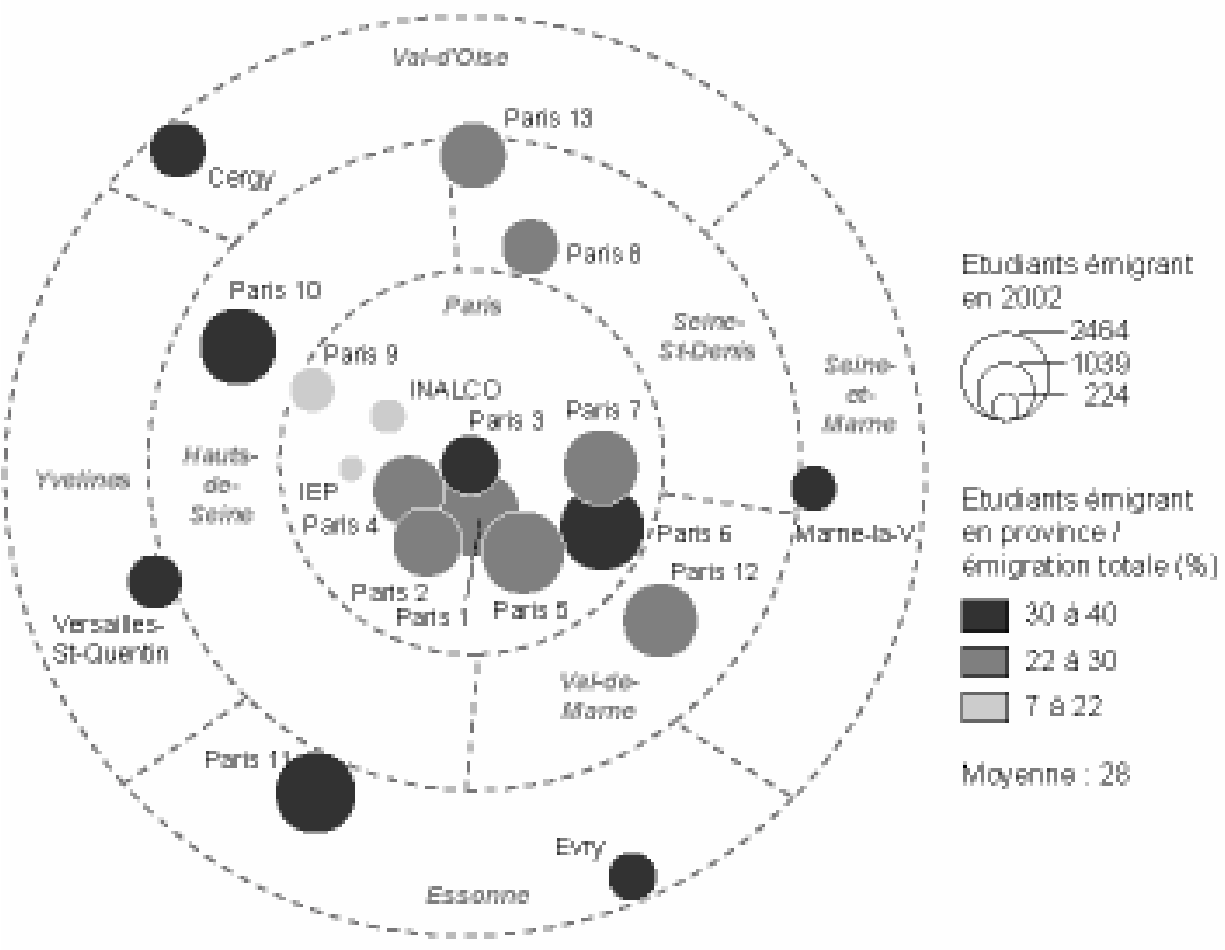

b) Les arrivées de province

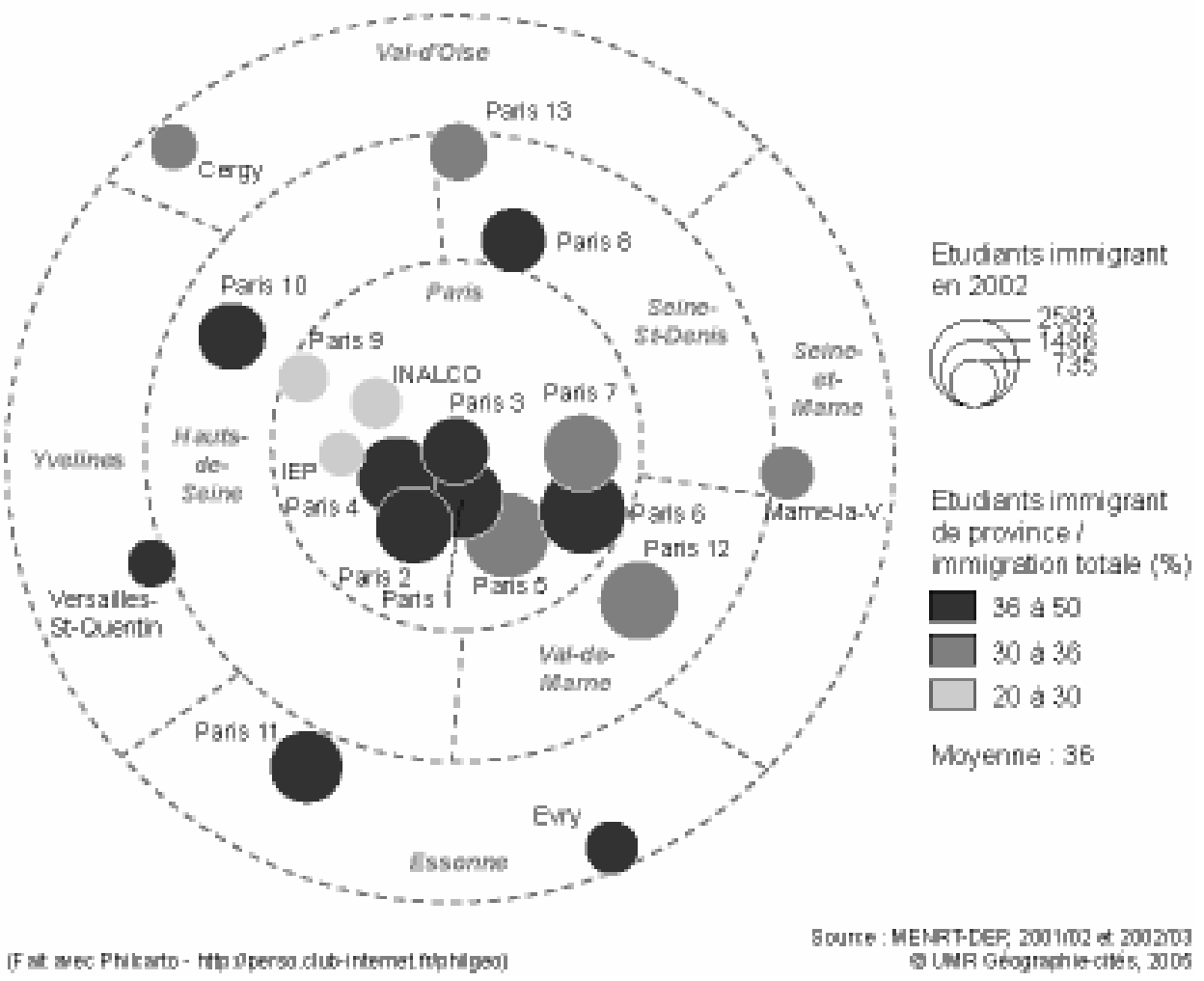




\section{Références}

Baron M. (1999), «Les équipements scolaires et universitaires », in Saint-Julien (dir.), Services et équipements, vol 10, p.54-59.

Berarida M., Lenglart D., Schlumberger-Guedj L. (1997), Etablissements d'enseignement supérieur et villes nouvelles d'Ile-de-France, 2 vol., Paris, Ministère de l'Education Nationale, de l'Enseignement Supérieur et de la Recherche, Ministère de l'Equipement, du Logement, des Transports et du Tourisme.

Berroir S., Cattan N., Saint-Julien Th. (2005), La contribution des villes nouvelles au polycentrisme francilien. Rapport de recherche, Paris, Ministère de l'Equipement.

Comité National d'Evaluation des établissements publics à caractère scientifique, culturel et professionnel (1996), Les universités nouvelles, Paris, CNE, 76 p.

Costes L. (2001), «Etudiants franciliens : inégalités dans la mobilité », Urbanisme, n³17, p.72-76.

DATAR (coord.) (1998), Développement universitaire et développement territorial. L'impact du plan U2 000 (1990-1995), Paris, La documentation française, coll. Informations et analyses.

Education et Formation (2004), L'enseignement supérieur grandes évolutions depuis 15 ans, $\mathrm{n}^{\circ} 67$.

Garnier M. (1995), Rapport sur les quatre universités nouvelles de la région Ile-de-France, Paris, CESRIF, 93 p.

IAURIF (2004), Les universités en Ile-de-France, Eléments d'analyse préalables à l'évaluation du Contrat de Plan, IAURIF, juillet.

Merlin P. (1994), «Les universités et les villes nouvelles de l’Ile-de-France », Annales de la recherche urbaine, $\mathrm{n}^{\circ} 62-63$, p. 206-213.

Ofipe résultats, 2005, «Les 4 universités nouvelles d'Ile-de-France ». Université de Marnela-Vallée, $\mathrm{n}^{\circ}$ 57-janvier, 8 p.

Ministère de l'Education Nationale, de l'enseignement supérieur et de la recherche, Direction de l'évaluation et de la prospective (2004), Atlas régional - les effectifs des étudiants en 2003-2004.

Raullin E., Saint-Julien Th. (dir.) (1998), La mobilité géographique des étudiants des universités, rapport de recherche MENRT-DATAR-CNRS.

Saint-Julien Th. (2003), « Les migrations des étudiants entre villes universitaires en France », in Ciattoni A. et Veyret Y. (dir.), Les fondamentaux de la géographie, Paris, Armand Colin. 